\title{
Editorial
}

\section{A PROPOSITO DE INTERVALO INTERGENESICO}

\section{Dr. Enrique Oyarzun Ebensperger}

Obstetricia y Ginecologia

\section{Clinica Universidad de los Andes}

En Chile, actualmente, las 3 mayores causas de muerte en los últimos años son enfermedades cardíacas, enfermedades cerebrovasculares y cáncer gástrico. La baja natalidad (13.4 nacidos vivos por año por cada 1000 habitantes el 2015) ha contribuido a la reducción de algunos eventos obstétricos adversos. Sin embargo, otros factores pueden contribuir al agravamiento de indicadores de salud materno infantil que han sido hasta ahora motivo de orgullo para nuestra especialidad. Ellos tienen que ver con el aumento de los embarazos a edades más tardías de la mujer, con el aumento de patologías medicas concomitantes con el embarazo, con el aumento de obesidad y diabetes, con el aumento de embarazos múltiples, con el aumento del uso de drogas, con la legalización del aborto, y con el aumento de las inmigraciones (crecimiento explosivo para los haitianos, por ejemplo, que entre 2005 y 2016 aumentaron $364.600 \%$ ).

En el contexto anterior, en aras de reducir factores de riesgo, y dado que es una pregunta frecuente, especialmente después de una pérdida reproductiva, resulta interesante el trabajo que esta revista trae de amigos mejicanos. El grupo del Hospital Ángeles Pedregal de Ciudad de México realiza una revisión de la literatura para contestar la pregunta de cuál es el intervalo intergenésico ideal para una nueva gestación.

El tiempo extremadamente breve o extremadamente largo entre una gestación y la siguiente se ha relacionado con complicaciones obstétricas. La definición sin embargo del intervalo entre embarazos o entre eventos obstétricos no es sencilla, y diferentes definiciones han intentado reducir el efecto de variables que pueden afectar los resultados buscados. Es así que si se evalúa el intervalo entre nacimientos, y el segundo nacimiento es prematuro, el intervalo será menor y estará asociado a mayor morbimortalidad neonatal, propia de la prematurez, y no secundaria al intervalo propiamente tal. Igualmente, si el primer parto es prematuro la probabilidad de prematurez en el siguiente embarazo aumenta y ello podría ser falsamente atribuido al intervalo entre gestaciones.

El intervalo óptimo entre embarazos no es claro y puede depender en parte del resultado del embarazo precedente. Después de un embarazo de término, con recién nacido vivo, la OMS ha recomendado un intervalo mayor de 2 años y menor de 5 , a partir de data que sugiere que intervalos bajo 18 meses se asocian a aumento del riesgo materno y perinatal (1, 2, 3, 4, 5). Los 2 años mínimos sugeridos serian consistentes con la recomendación de la UNICEF de 2 años de lactancia materna.

En mujeres de 35 años o más, 12 meses pueden ser suficientes si se considera que la fertilidad disminuye más tarde. Después de un aborto, en cambio, la recomendación tradicional ha sido esperar al menos 3 meses, pero la data disponible es conflictiva $(6,7,8$, 9). La OMS, basada en un meta análisis (10), sugiere esperar al menos 6 meses.

El trabajo de esta revista considera periodo intergenésico aquel que se encuentra entre la fecha del último evento obstétrico y el inicio del siguiente embarazo. Se sugiere como tiempo recomendado de espera para iniciar un siguiente embarazo mínimo 18 meses (período intergenésico corto, PIC) y no más de 60 meses (período intergenésico largo, PIL), para reducir el riesgo de eventos adversos maternos, perinatales y neonatales. El trabajo sigue entonces las recomendaciones de la OMS en esta materia.

El trabajo que comentamos se extiende también en los mecanismos fisiopatológicos involucrados en las consecuencias de un periodo intergenesico corto o largo ("depleción materna", "regresión fisiológica"), y en el tipo de sutura uterina y 
su relación con rotura uterina posterior, tema en particular que no corresponde al objetivo del trabajo, y para el cual no hay diferencias demostradas $(12,13)$. Su inclusión puede causar confusión en los lectores.

Es posible que una buena manera de evaluar los efectos del intervalo intergenesico sea considerar el tiempo transcurrido entre un nacimiento de término con recién nacido vivo y el inicio del siguiente embarazo. Debe recordarse, además, que más que cumplir un intervalo estricto es siempre más importante un control adecuado, en un sitio adecuado y con un especialista adecuado.

\section{REFERENCIAS}

1. Conde-Agudelo A, Rosas-Bermúdez A, KafuryGoeta AC. Effects of birth spacing on maternal health: a systematic review. Am J Obstet Gynecol 2007; 196:297.

2. Thiel de Bocanegra $H$, Chang R, Howell M, Darney $P$. Interpregnancy intervals: impact of postpartum contraceptive effectiveness and coverage. Am J Obstet Gynecol 2014; 210:311.e1.

3. Copen CE, Thoma ME, Kirmeyer S. Interpregnancy intervals in the United States: data from the birth certificate and the national survey of family growth. Natl Vital Stat Rep 2015; 64:1.

4. Conde-Agudelo A, Rosas-Bermudez A, Castaño F, Norton $\mathrm{MH}$. Effects of birth spacing on maternal, perinatal, infant, and child health: a systematic review of causal mechanisms. Stud Fam Plann 2012; 43:93.

5. Auger N, Daniel M, Platt RW, et al. The joint influence of marital status, interpregnancy interval, and neighborhood on small for gestational age birth: a retrospective cohort study. BMC Pregnancy Childbirth 2008; 8:7.

6. Ball SJ, Pereira G, Jacoby P, et al. Re-evaluation of link between interpregnancy interval and adverse birth outcomes: retrospective cohort study matching two intervals per mother. BMJ 2014; 349:g4333.

7. Davanzo J, Hale L, Rahman M. How long after a miscarriage should women wait before becoming pregnant again? Multivariate analysis of cohort data from Matlab, Bangladesh. BMJ Open 2012; 2.

8. Wong LF, Schliep KC, Silver RM, et al. The effect of a very short interpregnancy interval and pregnancy outcomes following a previous pregnancy loss. Am J Obstet Gynecol 2015; 212:375.e1.

9. Love ER, Bhattacharya S, Smith NC, Bhattacharya S. Effect of interpregnancy interval on outcomes of pregnancy after miscarriage: retrospective analysis of hospital episode statistics in Scotland. BMJ 2010; 341:c3967.

10. Kangatharan C, Labram S, Bhattacharya S. Interpregnancy interval following miscarriage and adverse pregnancy outcomes: systematic review and meta-analysis. Hum Reprod Update 2017; 23:221.

11. Marston, C. Report of a WHO technical consultation on birth spacing. World Health Organization, 2005; 1-37.

12. Caesarean section surgical techniques (CORONIS): a fractional, factorial, unmasked, randomised controlled trial. the Coronis collaborative group (including Enrique Oyarzun) The Lancet 2013; vol 382, issue 9888.pages 234248, July 20. doi:10.1016/SO140-6736(13)604419

13. Coronis. International study of caesarean section surgical techniques: the follow up study. The Coronis Collaborative Group. BMC Pregnancy and Childbirth 2013, 13:215 http://www.biomedcentral.com/1471-2393/13/215 